Check for updates

Cite this as: $B M J 2021 ; 372: n 858$ http://dx.doi.org/10.1136/bmj.n858 Published: 31 March 2021

\section{Update to living systematic review on drug treatments for covid-19}

This living systematic review by Siemieniuk and colleagues (BMJ 2020;370:m2980) has been updated. For the latest update, visit doi:. The latest version of this living systematic review includes results for new interventions angiotensin-converting enzyme inhibitors, anakinra, full dose anticoagulation, ivermectin, ivermectin plus doxycycline, JAK inhibitors, lopinavir-ritonavir plus interferon-beta, peginterferon lambda, proxalutamide, sulodexide, vitamin $\mathrm{C}$, and vitamin $\mathrm{D}$ (but certainty is generally low or very low); evidence that azithromycin may not have an impact on any patient-important outcome; evidence that interleukin- 6 inhibitors probably reduce mechanical ventilation (moderate certainty) and may reduce duration of hospitalisation (low certainty); evidence that JAK inhibitors probably reduce duration of mechanical ventilation (moderate certainty) and may reduce mortality (low certainty), mechanical ventilation (low certainty), and duration of hospitalisation (low certainty); evidence that colchicine may reduce mortality (low certainty) and mechanical ventilation (low certainty) in outpatients with non-severe disease; and evidence on ivermectin and ivermectin plus doxycycline, but whether they affect any patient-important outcome remains very uncertain. 